\title{
Interacciones multimodales en una clase de español de octavo año costarricense
}

Multimodal interactions in a Costa Rican eighth grade Spanish class

\author{
Volumen 21, Número 3 \\ Setiembre - Diciembre \\ pp. 1-33
}

\section{Carol Flores Solano}

\section{Citar este documento según modelo APA}

Flores Solano, Carol. (2021). Interacciones multimodales en una clase de español de octavo año costarricense. Revista Actualidades Investigativas en Educación, 21(3), 1-33. Doi. 10.15517/aie.v21i3.46284 


\title{
Interacciones multimodales en una clase de español de octavo año costarricense

\author{
Multimodal interactions in a Costa Rican eighth grade Spanish class
}

\section{Carol Flores Solano ${ }^{1}$}

Resumen: Esta investigación efectúa un análisis multimodal de los géneros empleados en la clase de español, por parte de una docente del Colegio Internacional Canadiense en Costa Rica, junto con los modos semióticos y sus metafunciones a lo interno de cada una de las fases genéricas. Es de enfoque cualitativo, de tipo descriptivo y corresponde a un estudio de caso. El corpus está constituido por las videograbaciones de la mediación pedagógica de la profesora dirigida a una población de catorce estudiantes de octavo año en dicho centro educativo. El análisis se centra, exclusivamente, en la figura de la docente y excluyó la información e intervenciones del estudiantado, a fin de garantizar su anonimato. Los resultados indican la presencia de cinco géneros: trabajo del estudiantado, revisión, lectura en conjunto, entrevista escolar y exposición docente. Como modos, se identificaron, de manera transversal, a todos ellos, la oralidad, escritura, recursos gráficos, gestos, movimientos corporales y desplazamiento. Como conclusiones, este estudio sugiere repensar la clase de español no como "típicamente lingüística", sino modalmente diversa, es decir, articulada por múltiples modos semióticos, todos los cuales se entrelazan durante la mediación. Además, sugiere que dicha etiqueta puede estar relacionada con el uso predominante de la escritura en las fases nucleares de los géneros.

Palabras clave: géneros, modos semióticos, multimodalidad, clase de español.

\begin{abstract}
This research carries out a multimodal analysis of the genres used in the Spanish class by a teacher at the Canadian International School in Costa Rica, together with the semiotic modes and their metafunctions within each of the generic phases. It is a qualitative, descriptive approach and corresponds to a case study. The corpus is constituted by the video recordings of the teacher's pedagogical mediation directed to a population of eight eighth grade students in this educational center. The analysis focuses exclusively on the figure of the teacher and excluded the participation of the students, in order to guarantee their anonymity. The results indicate the presence of five genres: student work, revision, joint reading, school interview and teacher exposition. As modes, orality, writing, graphic resources, gestures, body movements and displacement were identified as transversal to all of them. As conclusions, this study suggests rethinking the Spanish class not as "typically linguistic" but as modally diverse, that is, articulated by multiple semiotic modes, all of which intertwine during mediation. Furthermore, it suggests that such a label may be related to the predominant use of writing in the nuclear phases of the genres.
\end{abstract}

Key words: genres, semiotic modes, multimodality, Spanish class.

\footnotetext{
${ }^{1}$ Docente de la Universidad de Costa Rica en la Escuela de Estudios Generales en la Sede del Atlántico, Turrialba, Costa Rica. Máster en Lingüística de la Universidad de Costa Rica. Orcid https://orcid.org/0000-0003-3771-6339
}

Dirección electrónica: carol.flores@ucr.ac.cr

Artículo recibido: 19 de marzo, 2021

Enviado a corrección: 4 de junio, 2021

Aprobado: 16 de agosto, 2021 


\section{Introducción}

Tradicionalmente, la preeminencia histórica del sistema lingüístico en la producción del conocimiento (Kress, 2010; Kress y van Leeuwen, 2001) ha contribuido a la consolidación de dos mitos en torno a este último como recurso comunicativo (Manghi, 2011, p. 6): "por un lado, que todo lo que es posible de ser representado puede ser realizado por la lengua; y, segundo, que los demás recursos juegan un rol secundario y solo reiteran el significado lingüístico". Esta ha sido descrita como una visión "monomodal" de la representación y comunicación, y confiere a la lengua el estatus de recurso de significación por antonomasia (Kress, 2010).

Frente a esta perspectiva, la multimodalidad desvía el foco de atención de la lengua como recurso central y dominante, capaz de expresar todos los significados, y la ubica como un recurso entre otros para la semiosis, cuyos potenciales de significación deben relativizarse a la luz de los alcances de estos últimos (Kress, 2010). Este enfoque multimodal advierte sobre la multiplicidad de modos que se articulan tanto en los textos como en las interacciones comunicativas. A su vez, señala que la creación de significados ocurre no a través de uno, sino de los múltiples recursos disponibles en una cultura y contextos dados.

En ámbitos escolares, la multimodalidad es relevante, por cuanto advierte sobre los potenciales de significado de los diversos modos semióticos empleados en la enseñanza y el aprendizaje. Además, permite reconocer la naturaleza multimodal del conocimiento disciplinar y de la mediación docente, así como las particularidades de cada uno de estos recursos en la representación y la comunicación (Manghi, 2011). En este contexto, este estudio nace del interés por evidenciar esta naturaleza multimodal de la mediación docente en la clase de español, y subrayar la variedad de modos que se articulan en esta última e inciden en la construcción de los contenidos curriculares (Kress, Jewitt, Ogborn y Tsatsarelis, 2001).

Este trabajo constituye un estudio de caso y tiene como objetivo analizar, multimodalmente, el empleo de géneros en la clase de español, por parte de una docente de octavo nivel del Colegio Internacional Canadiense en Costa Rica en el año 2016. Pretende, en primer lugar, describir estructural y funcionalmente, los géneros mediante los cuales se construyen los contenidos curriculares; en segundo lugar, distinguir los modos semióticos empleados típicamente para cada género y sus etapas, así como las funciones que desempeñan y medios asociados a estas fases.

En el campo educativo, el análisis de los géneros es relevante, por cuanto evidencia que las interacciones diseñadas por el profesorado no son gratuitas ni azarosas, sino patrones relativamente regulares de significación, con una estructura clara y etapas con propósitos 
específicos, afianzados por su consistencia de uso en el entorno escolar. Además, visibiliza los significados creados a través de estas fases, en términos de cómo ocurre la modelación del contenido curricular, las relaciones entre participantes y vínculos internos entre etapas.

Por otro lado, el análisis de los modos es pertinente por dos motivos. En primer lugar, evidencia que los contenidos curriculares se modelan no solo genérica, sino multimodalmente, según los recursos de creación de significados disponibles en el contexto de clase. En segundo lugar, a partir de este acercamiento multimodal, este trabajo relativiza el carácter "típicamente lingüístico" atribuido a la clase de español y evidencia que la lengua coexiste con otros recursos de significación, también relevantes para la mediación en esta asignatura.

A excepción de Flores (2019), en Costa Rica, destaca la ausencia de estudios que describan multimodalmente las principales interacciones empleadas en la enseñanza de las asignaturas escolares, junto con las combinaciones multimodales típicas que despliegan en su realización. A nivel latinoamericano, existe un cuerpo consolidado de investigaciones que, desde el enfoque multimodal, ofrecen análisis de los géneros escolares; no obstante, ninguna de ellas se enfoca en la clase de español, sino en historia (Manghi, 2013a, 2013b; Manghi y Badillo, 2015; Manghi, Badillo y Villacura, 2014), biología (Manghi, 2013a; 2013b, Manghi y Córdova, 2011) y matemática (Manghi, 2013c; Manghi, 2010).

Todos los anteriores constituyen estudios de caso, cuyo corpus está conformado por grabaciones en video de uno a tres docentes por cada asignatura, quienes imparten sus lecciones a una población de secundaria. Además, guardan en común el hecho de centrarse en la figura del profesorado como diseñador de las interacciones (Kress, 2010), a fin de caracterizar genérica y multimodalmente los procesos de mediación facilitados por este último en contextos escolares específicos. Para mantener la coherencia con estas investigaciones y contrastar los resultados, este estudio se centró, exclusivamente, en la figura de la docente y en el análisis multimodal de su mediación pedagógica.

De acuerdo con estas investigaciones, en la clase de historia, se identificaron dos géneros comunes a todos los estudios de caso, denominados relato histórico y explicación de las causas (Manghi, 2013a, Manghi, Badillo y Villacura, 2014, Manghi y Badillo, 2015). Adicionalmente, en casos particulares, se identificaron los géneros metahistórico, modelamiento de interpretación histórica y procedimental (Manghi, Badillo y Villacura, 2014). Todos ellos guardan en común el llevarse a cabo mediante articulaciones multimodales de oralidad, escritura, gestos y recursos gráficos, como tablas, dibujos o mapas, predominantemente, a través de la interacción cara a cara y el uso de la pizarra. 
En la clase de biología, destacan como géneros las definiciones y las explicaciones secuenciales (Manghi y Córdova, 2011, Manghi, 2013a). Las primeras tienen como objetivo exponer los caracteres genéricos y diferenciales de entidades, y se categorizaron en tres tipos: descriptivas, clasificatorias y composicionales (Manghi, 2013a). El segundo formula ejemplos en la vida diaria de procesos naturales, los cuales familiarizan al estudiantado con los fenómenos y motivan su explicación científica (Manghi, 2013a).

En ambos géneros, los dibujos de esquemas estructurales resultaron ser el recurso semiótico en común y más relevante (Manghi, 2013a; Manghi y Córdova, 2011), debido a las particularidades de su discurso científico y su potencial de significado visual para la conceptualización (Lemke, 1998). Estos se emplean a manera de una definición visual, que informa sobre la morfología de las estructuras y/o procesos biológicos. Sus significados y relaciones se modulan a través de la oralidad y los gestos, en la interacción cara a cara (Manghi y Córdova, 2011).

En la clase de matemática, se identificó el microgénero modelaje de procedimiento (Manghi, 2013c), el cual muestra al estudiantado la manera de desarrollar un procedimiento a través de diferentes ejemplos concretos (Manghi, 2013c). Este suele introducirse mediante una fase previa denominada lectura del informe, la cual constituye el punto de partida para el ejemplificar su resolución. Esta fase forma parte de un microgénero distinto llamado descripción del problema (Manghi, 2013c), el cual se registró, también, en otros momentos de la interacción, con el propósito enunciar en voz alta el problema matemático y caracterizarlo lingüísticamente. Finalmente, se identificó el género análisis de razonamiento, cuyo objetivo consiste en comentar para el estudiantado los procedimientos desarrollados previamente en el pizarrón (Manghi, 2010).

En matemática, todos los géneros identificados se efectúan, por lo general, a través de una articulación de oralidad, gestos deícticos y escritura en el pizarrón. Precisamente, la lengua - oral y escrita - se utiliza como un metalenguaje, para definir y reelaborar los términos técnicos de la disciplina (Manghi, 2013c). Además, la enseñanza se caracteriza por desplegar temporalmente el discurso y transformar las representaciones a través de mecanismos de transducción semiótica (Manghi, 2013c).

En el marco de las investigaciones anteriores, ante la ausencia de estudios para la clase de español, esta investigación pretende contribuir a las caracterizaciones ya realizadas para las disciplinas de historia, biología y matemática. Además, procura robustecer los hallazgos ya disponibles en otros contextos latinoamericanos sobre los géneros desplegados por el 
profesorado, así como las particularidades modales que distinguen cada uno de ellos y que caracterizan la construcción de los contenidos curriculares.

\section{Referente teórico}

\subsection{Multimodalidad, modos y medios}

La multimodalidad puede concebirse como una perspectiva o enfoque de análisis de la comunicación y representación, que advierte sobre la presencia de múltiples recursos para la creación de significados (Kress, 2010; Kress y van Leeuwen, 2001). Desde esta visión, las culturas modelan una amplia gama de modos, con el fin satisfacer los intereses sociales de las distintas comunidades (Kress et al., 2001). El empleo de diferentes modos implica, en primer lugar, que los significados se elaboran de forma distinta en cada uno de ellos y no se comprenden de la misma manera por todos los intérpretes. En segundo lugar, sugiere que los significados lingüísticos - realizados ya sea mediante el habla o la escritura - se entrelazan con otros creados en modos distintos en el contexto comunicativo y esta interacción, en sí misma, produce significado (Manghi, 2011; Kress et al., 2001)

Los modos se definen como sistemas para la creación de significados, compuestos por diferentes recursos semióticos (Kress et al., 2001). Los recursos semióticos se enmarcan dentro del modo y se definen como las posibilidades de representación a lo interno de cada sistema que, relacionadas con su materialidad y regularidades de empleo social, permiten crear significados (Bezemer y Kress, 2008). Así, posibilitan satisfacer las necesidades representacionales y comunicativas de los creadores de signos (Kress, 2010; Kress y van Leeuwen, 2001; van Leeuwen, 2005).

Por ejemplo, la escritura, en tanto modo, posee como recursos "la sintaxis, la gramática, el léxico, recursos gráficos como el tipo de fuente y de composición como la puntuación". Incluso, puede emplear el color. En contraste, el habla, en tanto modo, "posee como recursos modales la intensidad, timbre y variaciones de timbre, cualidad vocal/tonal, extensión y silencios" (Bezemer y Kress, 2008, p. 7).

A partir de las propuestas de Cope y Kalantzis (2009), Kress et al. (2001) y Lemke (1990), los modos pueden organizarse en tres sistemas semióticos: lingüístico, visual y accional, los cuales se resumen en la Tabla 1. 


\section{Tabla 1}

\section{Sistemas semióticos y modos correspondientes}

\begin{tabular}{cl}
\hline Sistema lingüistico & lengua oral y escrita. \\
\hline Sistema visual & $\begin{array}{l}\text { Imágenes, ilustraciones, dibujos, figuras, tablas, cuadros, gráficos, mapas y } \\
\text { presentaciones audiovisuales especializadas. }\end{array}$ \\
\hline Sistema accional & $\begin{array}{l}\text { Gestos, posturas, usos del cuerpo, movimientos del cuerpo o miembros de } \\
\text { este, desplazamientos en el espacio y empleo de este último. }\end{array}$ \\
\hline
\end{tabular}

Fuente: Elaboración propia con base en Cope y Kalantzis (2009), Kress et al. (2001) y Lemke (1990).

La noción de modo conduce, a su vez, a la de textos multimodales. Estos se definen como aquellos compuestos por dos o más modos semióticos, y cuyos significados se realizan mediante la articulación de estos últimos (Bezemer y Kress, 2008; Kress, 2010; Kress y van Leeuwen, 2001; Manghi, 2011; van Leeuwen, 2005). Cabe destacar que la noción de texto multimodal trasciende el medio impreso y modo lingüístico, e incluye como entidades textuales las prácticas sociales (van Leeuwen, 2005).

De acuerdo con esta definición, la actuación docente puede ser interpretada como texto multimodal, compuesto en sí mismo por distintos modos semióticos, por ejemplo, los gestos, los movimientos corporales, la postura, los desplazamientos por el espacio, entre otros (Lemke, 1990; Kress et al., 2001). Esta actuación, a su vez, se articula con los demás recursos - diferentes de la corporalidad del profesorado - disponibles en el contexto de clase, todos los cuales convierten las interacciones escolares en un texto multimodal macro.

Por otra parte, desde la perspectiva multimodal, toda práctica social desempeña las tres metafunciones de creación de significados - representacional, interpersonal y textual -, a partir de las cuales es posible identificar estos últimos (Halliday, 1982; Kress, 2010). La Tabla 2 describe estas metafunciones, así como su aplicación para el análisis de las interacciones escolares (Kress et al., 2001).

Tabla 2

Metafunciones de creación de significados y su aplicación al ámbito educativo

\begin{tabular}{ccc}
\hline Metafunción & Descripción & Aplicación educativa \\
\hline Representacional & $\begin{array}{c}\text { Construye la realidad en términos de } \\
\text { acciones, participantes, estados y eventos. }\end{array}$ & $\begin{array}{c}\text { Construye los contenidos curriculares en } \\
\text { términos de entidades que se involucran y } \\
\text { se relacionan con procesos. }\end{array}$ \\
\hline Interpersonal & $\begin{array}{c}\text { Establece las relaciones sociales y de } \\
\text { poder entre aquellos que interactúan en la } \\
\text { comunicación. }\end{array}$ & $\begin{array}{c}\text { Establece las relaciones sociales, de poder } \\
\text { y conocimiento, así como los agentes en la } \\
\text { interacción. }\end{array}$ \\
\hline Textual & $\begin{array}{c}\text { Forma textos como entidades semióticas } \\
\text { complejas, los cuales poseen coherencia y y } \\
\text { cohesión interna y con su entorno. }\end{array}$ & $\begin{array}{c}\text { Determina los vínculos entre las diversas } \\
\text { fases y dota de coherencia a la actividad } \\
\text { didáctica. }\end{array}$ \\
\hline
\end{tabular}

Fuente: Elaboración propia con base en Halliday (1982), Kress (2010) y Kress et al. (2001). 
Por último, cabe destacar que los modos se realizan en los medios semióticos, a través de los cuales encuentran distintas materializaciones. Los medios se definen como las sustancias físicas de ejecución, modeladas culturalmente, como 'superficies' para instanciar los modos (Kress y van Leeuwen, 2001). Estos constituyen la dimensión orgánica y material en cada modo se produce de forma concreta y difunde (Bezemer y Kress, 2008). Dentro de la categoría de medios, se incluyen recursos o superficies físicas, analógicas y digitales, por ejemplo, interacciones cara a cara, papeles, cuadernos, objetos, pizarra, el computador, grabaciones, altavoces, parlantes, proyector, entre otros.

\subsection{Géneros}

Los géneros se definen como configuraciones recurrentes de significado que representan las prácticas sociales de una cultura dada (Martin y Rose, 2008). Son procesos sociales, ya que involucran la creación de significados en interacción; se orientan hacia un objetivo, que es responsable de su organización estructural, y se organizan en etapas, con el propósito de alcanzar esa meta (Martin y Rose, 2008). Los géneros se experimentan como prácticas reconocibles, es decir, como constituidas por acciones relativamente regulares y estables en el contexto social (Kress, 2010; van Leeuwen, 2005).

Los modos constituyen los recursos semióticos culturales para realizar los géneros. Estos últimos instancian los discursos en situaciones comunicativas dadas, mediante formas de interacción social específicas, a través de los modos y medios disponibles. Dicho de otra manera, los géneros constituyen el estrato intermedio de la interacción, que modela los discursos sociales en configuraciones recurrentes de significado y los realiza a través de los modos y medios semióticos (Kress, 2010; van Leeuwen, 2005).

En contextos educativos, Martin y Rose (2008, p. 139) distinguen cuatro familias de géneros a través de las cuales se construye el discurso científico: 1. Informes 2. Procedimientos 3. Explicaciones y 4. Recuentos. Por motivos de brevedad y debido a que los resultados de esta investigación corresponden a las dos primeras familias, estas son las únicas explicadas en este apartado. A fin de profundizar en las demás, se recomienda revisar a los autores.

Los géneros procedimentales se caracterizan por dirigir una secuencia de actividades en relación con ciertos objetos y ubicaciones explícitos, para obtener efectos o productos semióticos particulares enunciados a su inicio. La interacción dicta una secuencia de acciones especializada con un orden lógico y temporal, para cuya ejecución designa recursos, 
locaciones e información específica en el contexto de los agentes. Estos géneros constituyen textos pedagógicos y cumplen una función especializada, instrumental o ritual en la cultura, por cuanto acercan un conocimiento a participantes que, en principio, lo desconocen, a través de la mentoría de un experto (Martin y Rose, 2008).

Dentro de los géneros procedimentales, se distinguen los procedimientos cotidianos y cooperativos (Martin y Rose, 2008). Los primeros, por lo general, se desarrollan en interacciones de uno a uno y requieren la presencia simultánea del mentor y aprendiz durante la ejecución del procedimiento, de modo que este último pueda ajustarse continuamente y coincida con la condición particular de cada paso de la actividad. Los procedimientos cooperativos requieren más de un agente para llevarse a cabo, cada uno de los cuales debe ser identificado con claridad, así como las herramientas, tareas y la porción del entorno sobre la cual actúa. Por tanto, suelen explicitar los individuos y objetos al inicio de cada instrucción, y acompañarse de especificaciones sobre sus actuaciones.

Los géneros informativos representan, discursivamente, fenómenos y entidades a partir de una descripción de sus propiedades, clasificación taxonómica o composición (Martin y Rose, 2008). A diferencia de los procedimentales, no enfatizan la acción de sujetos respecto de ciertos objetos y ubicaciones; sino que se enfocan en las entidades, en las actividades que llevan a cabo o en las que participan. Así, suelen emplear estructuras de causa y efecto que establecen relaciones lógicas entre los fenómenos, los procesos en los que se involucran y rasgos que las definen (Martin y Rose, 2008).

Por otra parte, como se dijo antes, cada género está conformado por etapas, las cuales constituyen su secuenciación típica (Martin y Rose, 2008). Estas fases desempeñan acciones funcionales, es decir, cumplen finalidades particulares dentro de la secuencia (Lemke, 1990). El objetivo del género se alcanza al cumplir las funciones particulares de sus etapas constituyentes. Además, estas fases lo diferencian de otras interacciones dentro de una misma familia genérica, y le confieren su especificidad estructural y funcional.

Por último, cabe aclarar que, en el ámbito escolar, las funciones de las etapas no pueden disociarse del contexto educativo en que se enmarcan los géneros, ya que se encuentran permeadas las finalidades pedagógicas de este último (Kress et al., 2001). De esta forma, cada fase puede describirse, primero, en términos de las Actividades Típicas de Aula que la caracterizan (en adelante ATA) (Sánchez, García, Rosales, de Sixte y Castellano, 2008), las cuales pueden explicarse en términos funcionales y modales. Esta exposición permite vincular 
la interacción escolar con su análisis multimodal y explicar las particularidades a lo interno de las etapas en relación con las actividades pedagógicas.

\section{Metodología}

\subsection{Enfoque}

Esta investigación es de enfoque cualitativo, de tipo descriptivo y corresponde a un estudio de caso. Se llevó a cabo con una profesora de secundaria de la asignatura de español, la cual se ofreció voluntariamente para la investigación y cuya participación fue aceptada según criterios de conveniencia, oportunidad y disponibilidad (Quintana-Peña, 2006). Puesto que ella trabajaba en el Colegio Internacional Canadiense, este fue el centro educativo en donde se realizó el estudio. La profesora trabajó con el único grupo de estudiantes de octavo nivel del centro educativo e inició sus labores en la institución en el ciclo lectivo del 2016, año en el cual se grabó el corpus de investigación. El grupo contaba con un total de catorce estudiantes, cuyas edades rondaban entre los 13 y 14 años. La docente y el estudiantado firmaron un consentimiento informado previamente a su participación.

\subsection{Unidades de análisis}

El corpus de investigación está constituido por las grabaciones en video de la mediación pedagógica de cuatro unidades curriculares de la asignatura de español y sus correspondientes sesiones (Sánchez et al., 2008), las cuales se detallan en la Tabla 3.

Tabla 3

Corpus de investigación

\begin{tabular}{|c|c|c|c|c|}
\hline $\begin{array}{c}\text { Fechas de observación y } \\
\text { grabación }\end{array}$ & Unidad curricular & $\begin{array}{l}\text { Lengua/ } \\
\text { Literatura }\end{array}$ & $\begin{array}{l}\text { Número total de } \\
\text { sesiones }\end{array}$ & $\begin{array}{c}\text { Tiempo total de } \\
\text { clases }\end{array}$ \\
\hline $\begin{array}{c}30 \text { de mayo, } 31 \text { de mayo, } 1 \\
\text { de junio de } 2016\end{array}$ & $\begin{array}{l}\text { Aire Frío - H.P. } \\
\text { Lovecraft }\end{array}$ & Literatura & 3 sesiones & 128 minutos \\
\hline 6 de junio de 2016 & Español de Costa Rica & Lengua & 1 sesión & 42 minutos \\
\hline $\begin{array}{c}7 \text { de junio, } 8 \text { de junio de } \\
2016\end{array}$ & Español de América & Lengua & 2 sesiones & 92 minutos \\
\hline $\begin{array}{c}9 \text { de junio, } 10 \text { de junio de } \\
2016\end{array}$ & $\begin{array}{l}\text { La Calera - Carlos } \\
\text { Salazar Herrera }\end{array}$ & Literatura & 2 sesiones & 87 minutos \\
\hline Total & & & 8 sesiones & 349 minutos \\
\hline
\end{tabular}

Fuente: Elaboración propia (2021).

\subsection{Técnicas de recolección}

Como técnicas de recolección, se empleó la observación no participante, así como grabaciones en video de las interacciones de clase observadas. La Tabla 3 indica las unidades 
curriculares, las fechas de las grabaciones y el tiempo total que tardó su enseñanza y que fue registrado. Cabe destacar que el criterio para la grabación fue curricular, no temporal. Es decir, una vez obtenido el acceso al aula, se buscó registrar dos contenidos curriculares distintos de lengua y literatura, independientemente del número de lecciones requeridos para enseñarlos, y no grabar un mínimo de minutos de clase.

Para las grabaciones, se empleó una sola cámara de video, marca Sony, modelo HDRXR160, la cual se ubicó en la sección posterior del aula, de espaldas al estudiantado. Desde esta posición, tuvo como foco la actuación de la docente y se empleó para registrar, de forma articulada y simultánea, las diversas formas de interacción - géneros -, los tres sistemas semióticos y modos asociados - lingüístico, visual y accional (Kress et al., 2001) - y los medios empleados por la profesora durante la enseñanza.

Pese a que el estudiantado apareció de espaldas a la cámara en las grabaciones, durante mediación pedagógica e interacciones con la docente; toda su información e intervenciones se excluyeron de los resultados. Esta investigación se enfocó, exclusivamente, en la figura de la profesora a fin de caracterizar la representación y comunicación de los contenidos curriculares en este contexto particular. Como se mencionó antes, esta decisión guarda simetría con la metodología de investigaciones anteriores, las cuales se centran en los procesos de mediación facilitados por profesorado. Asimismo, responde a la intención de garantizar el anonimato del estudiantado.

\subsection{Procesamiento y análisis}

La sistematización y análisis de los datos se llevaron a cabo en cinco etapas, mediante un instrumento original de la investigadora y sin el apoyo de software en ninguna fase. Este instrumento corresponde a la Tabla 4.

En la primera etapa, en correspondencia con la Tabla 3, las distintas unidades curriculares se segmentaron en cada una de sus sesiones (Ver Tabla 4, sombreado gris). En la segunda etapa, cada sesión se segmentó en las distintas Actividades Típicas de Aula (ATA) (Sánchez et al, 2008), es decir, en las diversas actividades de mediación diseñadas por la docente para impartir los contenidos. Es fundamental destacar que, en este punto, el análisis se centró en determinar, únicamente, estas dinámicas de clase y no los géneros, cuya identificación se realizó en una etapa posterior. En la columna de ATA (Tabla 4), se nombró cada actividad identificada con un nombre alusivo, propuesto por la investigadora. 
En la tercera etapa, las ATA identificadas en la fase anterior se segmentaron en cada una de las etapas mediante las cuales se llevaron a cabo (Tabla 4). Para este fin, se empleó el catálogo de estructuras de actividades (Lemke, 1990), el cual permitió reconocer fases típicas en las interacciones de clase según sus funciones específicas. En la columna de etapas (Tabla 4), se nombró cada fase con un nombre original de la investigadora. Además, debido a que la interacción se analizó desde la perspectiva multimodal, en la columna de descripción (Tabla 4), se empleó una captura de pantalla, a fin de representar de forma visual y no verbal las actividades, los vínculos entre participantes, modos y medios en cada fase.

Una vez segmentadas las etapas de las ATA, las columnas de modos y medios (Tabla 4), se emplearon para identificar, respectivamente, los recursos de creación de significados y superficies materiales de producción o distribución, empleados para resolver las actividades de mediación en cada fase (Lemke, 1998; Kress y van Leeuwen 2001, Kress, 2010).

Tabla 4

Instrumento de sistematización y análisis de datos Unidad curricular: Español de Costa Rica. Sesión: 1

\begin{tabular}{|c|c|c|c|c|c|c|}
\hline Tiempo & ATA & Género & Etapas & Descripción & Modos & Medios \\
\hline 00:00 & Canción & Trabajo del & Organización & & $\begin{array}{l}\text { oralidad, gestos, } \\
\text { mirada, } \\
\text { movimientos de } \\
\text { manos y brazos, } \\
\text { desplazamiento } \\
\text { por el espacio, } \\
\text { uso de objetos }\end{array}$ & $\begin{array}{l}\text { Interacción } \\
\text { cara a cara, } \\
\text { parlantes }\end{array}$ \\
\hline $00: 58$ & & & Instrucciones & & $\begin{array}{l}\text { oralidad, gestos, } \\
\text { mirada, } \\
\text { movimientos de } \\
\text { manos y brazos, } \\
\text { uso de objetos }\end{array}$ & $\begin{array}{l}\text { Interacción } \\
\text { cara a cara, } \\
\text { parlantes, } \\
\text { celular }\end{array}$ \\
\hline
\end{tabular}

Fuente: Elaboración propia con fotografías de archivo personal (2021).

En la cuarta etapa, una vez detalladas las ATA, etapas, modos y medios, se procedió a identificar patrones regulares, conformados por bloques con las mismas etapas y que se repitieron en diferentes momentos de interacción. En este punto, la Tabla 4 permitió constatar la existencia de un gran número de ATA en apariencia "distintas", las cuales, no obstante, desarrollaban interacciones recurrentes, de la misma naturaleza. Este hallazgo permitió identificar los géneros e hizo posible completar esta columna en el instrumento (Tabla 4). Cada uno de ellos recibió un nombre distinto, original de la investigadora.

La etapa final del análisis requirió la clasificación de los géneros según la tipología de Martin y Rose (2008) a fin de identificar los propósitos que cumplen en el contexto escolar. 
Además, se identificó el contenido curricular asociado, cada una de sus etapas, y los modos y medios más recurrentes en estas últimas. Finalmente, para determinar las funciones de las etapas genéricas y de los modos, se analizó el cumplimiento de las metafunciones representacional, interpersonal y textual (Halliday, 1982; Kress, 2010).

\section{Resultados}

La Tabla 5 detalla los géneros identificados en esta investigación, su tipología, etapas y contenido curricular asociado.

Tabla 5

Géneros identificados en clase de español

\begin{tabular}{|c|c|c|c|c|c|c|c|}
\hline \multicolumn{2}{|c|}{$\begin{array}{l}\text { Tipo de } \\
\text { género }\end{array}$} & \multirow{2}{*}{$\begin{array}{c}\text { Género } \\
\begin{array}{c}\text { Trabajo de } \\
\text { los }\end{array} \\
\text { estudiantes }\end{array}$} & \multicolumn{4}{|c|}{ Etapas } & \multirow{2}{*}{$\begin{array}{c}\begin{array}{c}\text { Contenido } \\
\text { curricular } \\
\text { asociado }\end{array} \\
\\
\text { Lengua y } \\
\text { literatura }\end{array}$} \\
\hline \multirow{4}{*}{ 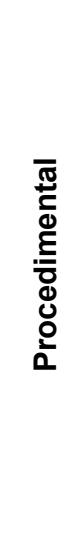 } & $\begin{array}{l}\stackrel{0}{\frac{\pi}{\sigma}} \\
\frac{0}{0} \\
\frac{0}{0} \\
ن\end{array}$ & & $\begin{array}{c}1 . \\
\text { Instrucciones }\end{array}$ & $\begin{array}{c}2 . \\
\text { Organización }\end{array}$ & $\begin{array}{l}\text { 3. Trabajo } \\
\text { de mesa }\end{array}$ & 4. Revisión. & \\
\hline & \multirow{3}{*}{ 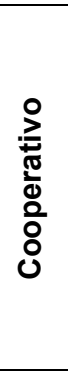 } & $\begin{array}{l}\text { Lectura en } \\
\text { conjunto }\end{array}$ & $\begin{array}{c}1 . \\
\text { Instrucciones } \\
\end{array}$ & $\begin{array}{c}2 . \\
\text { Organización }\end{array}$ & $\begin{array}{l}\text { 3. Lectura } \\
\text { grupal }\end{array}$ & $\begin{array}{l}\text { 4. Valoración } \\
\text { personal }\end{array}$ & Literatura \\
\hline & & Revisión & $\begin{array}{c}1 . \\
\text { Instrucciones }\end{array}$ & $\begin{array}{c}2 . \\
\text { Organización }\end{array}$ & $\begin{array}{l}\text { 3. Trabajo } \\
\text { grupal }\end{array}$ & $\begin{array}{c}4 . \\
\text { Verificación } \\
\text { del trabajo en } \\
\text { grupo } \\
\end{array}$ & Lengua \\
\hline & & Entrevista & $\begin{array}{c}1 . \\
\text { Instrucciones }\end{array}$ & $\begin{array}{c}2 . \\
\text { Organización }\end{array}$ & $\begin{array}{c}2 . \\
\text { Redacción } \\
\text { de } \\
\text { preguntas }\end{array}$ & $\begin{array}{l}\text { 3. Diálogo } \\
\text { con el } \\
\text { entrevistado }\end{array}$ & Lengua \\
\hline \multicolumn{2}{|c|}{ Informativo } & $\begin{array}{l}\text { Exposición } \\
\text { docente }\end{array}$ & $\begin{array}{c}1 . \\
\text { Conocimientos } \\
\text { previos }\end{array}$ & $\begin{array}{l}\text { 2. Exposición } \\
\text { docente }\end{array}$ & 3. Síntesis & & $\begin{array}{l}\text { Lengua y } \\
\text { literatura }\end{array}$ \\
\hline
\end{tabular}

Fuente: elaboración propia (2021).

De acuerdo con la Tabla 5, se registró una mayor variedad de géneros procedimentales (4) en contraste con un único género informativo. Los primeros son, además, estructuralmente más extensos, pues registran, en todos los casos, cuatro etapas; a diferencia del segundo que cuenta con tres. Este hallazgo sugiere que la mediación en la clase de español no ocurre, únicamente, mediante la explicación magistral, sino que esta alterna con una serie de procedimientos empleados para construir los contenidos curriculares de formas diversas.

Por otra parte, en los géneros procedimentales, destaca la recurrencia de las fases de instrucciones y organización. Posterior a ellas, las dos últimas etapas presentan una mayor divergencia entre géneros, lo cual contribuye a su especificidad estructural y funcional propia. Las fases del género informativo son exclusivas y no se repiten en ninguna otra interacción. 
Cada género se construyó en torno a una fase nuclear, cuya presencia es común a todas las interacciones y típica de su configuración (Tabla 5. Ver sombreado gris). Esta noción de "núcleo del género" resultó pertinente, en primer lugar, debido a su recurrencia y a la regularidad de las funciones que realiza. En segundo lugar, puesto que no se ha visto desarrollada en las investigaciones anteriores, lo cual resaltó su pertinencia como un aporte de este estudio.

Como núcleo del género se denominó la etapa genérica central, que se encarga de asignar prácticas de reelaboración discursiva. Estas últimas se definen como interacciones específicas, que tienen como objetivo resignificar los contenidos curriculares mediante la acción semiótica (Kress, 2010) e interpretaciones del estudiantado. Estas prácticas pueden manifestarse como la ejecución de procedimientos (en los géneros procedimentales) o como la construcción de entidades semióticas (en el género informativo), y comprenden tanto las interacciones como los instrumentos empleados para estos fines.

La presencia de un núcleo en las interacciones se explica, en primer lugar, por el contexto escolar. El objetivo de un género no es solo "procedimental" o "informativo", sino educativo, en el sentido de que busca el aprendizaje de ciertos contenidos curriculares por parte de una audiencia (Kress et al., 2001). Esta finalidad recae en el núcleo, donde el estudiantado suele resolver tareas específicas a fin de aprehender estos últimos. En segundo lugar, el núcleo del género presentó una configuración particular de las tres metafunciones, que fue recurrente en todos casos y lo caracterizó como tal. Esta configuración se empleó como criterio para identificar la fase nuclear y muestra los siguientes rasgos distintivos, independientemente del género:

a. Representacionalmente, el núcleo se define por asignar las prácticas de reelaboración discursiva, las cuales concentran el trabajo de modelación y reinterpretación del contenido curricular. Como resultado, esta fase desarrolla la mayor parte la creación semiótica, precisamente, en respuesta a las tareas asignadas.

b. Interpersonalmente, el núcleo confiere el mayor protagonismo al estudiantado, quien se encarga de resolver las prácticas de reelaboración asignadas ${ }^{2}$. Su protagonismo es un rasgo inherente a esta etapa y responde a la finalidad pedagógica de los géneros, según la cual, se motiva al estudiantado a crear su propio conocimiento.

2 El género exposición docente no confiere a esta predominancia al estudiantado en la fase nuclear. Esta particularidad se explicará más adelante, al describir dicho género. 
c. Textualmente, todas las fases de los géneros se organizan en torno a la fase nuclear, con el propósito de anticipar las prácticas de reelaboración discursiva que esta desarrolla o remitir a los significados construidos en ella, al finalizar la interacción. Como resultado, el núcleo reúne las funciones de las fases anteriores y posteriores - las condensa -, ya que está al servicio de la modelación del discurso y encierra la mayor parte de los significados elaborados.

\subsection{Géneros procedimentales: trabajo del estudiantado, revisión, entrevista y lectura en conjunto}

De acuerdo con Martin y Rose (2008), el género trabajo del estudiantado se definió como un procedimiento cotidiano y se empleó indistintamente tanto para contenidos curriculares de lengua como de literatura. Es decir, constituyó un patrón de creación de significados general, para discursos escolares de diversa naturaleza en clase de español.

Los géneros de revisión, lectura en conjunto y entrevista se clasificaron como procedimientos cooperativos, por cuanto su ejecución exigió la participación grupal, simétrica y activa de todos los miembros del estudiantado. Precisamente, esta particularidad en su metafunción interpersonal categorizó como cooperativas las tres interacciones y, al mismo tiempo, las distinguió del género trabajo del estudiantado, cuyos procedimientos se realizan, predominantemente, de manera individual. Además, su presencia sugiere que la clase de español no favorece una modalidad interpersonal en particular, sino que balancea la mediación a través trabajo individual y grupal entre participantes.

Los hallazgos anteriores difieren de los encontrados para la clase de historia (Manghi, Badillo y Villacura, 2014), que identificaron el género procedimental como un tipo de transición entre los géneros relato histórico y explicación de las causas, cuyo fin consiste en introducir el próximo género mediante una contextualización discursiva y explicitación de la tecnología. En la clase de español, los géneros procedimentales no constituyen estructuras de transición, sino una categoría amplia de interacciones, que, no obstante, guarda en común con la primera su naturaleza directiva y secuencial (Martin y Rose, 2008).

Por otra parte, pese a su configuración interpersonal en común, los géneros cooperativos se distinguen entre sí debido a sus etapas, funciones representacionales, textuales y respecto del contenido curricular en función del cual se utilizan. Así, la revisión y la entrevista se identificaron, únicamente, para contenidos de lengua; la lectura en conjunto, solo para 
literatura. Esta es otra diferencia relevante entre dichos géneros y el trabajo del estudiantado, el cual se empleó para ambos tipos de contenidos curriculares.

Dentro de los géneros cooperativos, la revisión muestra ciertas particularidades que la diferencian del resto de interacciones. En primer lugar, esta se inserta, típicamente, luego del trabajo del estudiantado, como cuarta etapa del género y con el objetivo de verificar los significados construidos en este último (ver Tabla 6, sombreado amarillo). En esta posición, funciona como la etapa ulterior del género que lo precede, el cual le provee el contenido curricular por valorar.

Por otra parte, la revisión muestra una estructura con fases genéricas propias, distintas de las del trabajo del estudiantado (ver Tabla 6, sombreado verde), que resignifican y cotejan los significados construidos en este último, a través de nuevos procesos. Finalmente, se debe recordar que la revisión posee una configuración interpersonal colectiva, que la diferencia el primero y la categorizan como procedimiento cooperativo. Por los motivos anteriores - su función representacional particular, su estructura genérica propia y carácter cooperativo - la revisión se distinguió como un género por separado.

Finalmente, si bien se utiliza después del trabajo del estudiantado, la revisión no aparece en todas las ocasiones en que este se registra. Su instanciación es, en última instancia, optativa y depende del uso de una nueva actividad pedagógica para evaluar los resultados del género anterior. En ese sentido, la revisión está sujeta a las decisiones de la docente como diseñadora y de si esta evalúa los resultados del primer procedimiento mediante otra interacción, la cual termina por constituir un nuevo género.

La Tabla 6 ilustra, multimodalmente, las etapas de los géneros procedimentales. 
Tabla 6

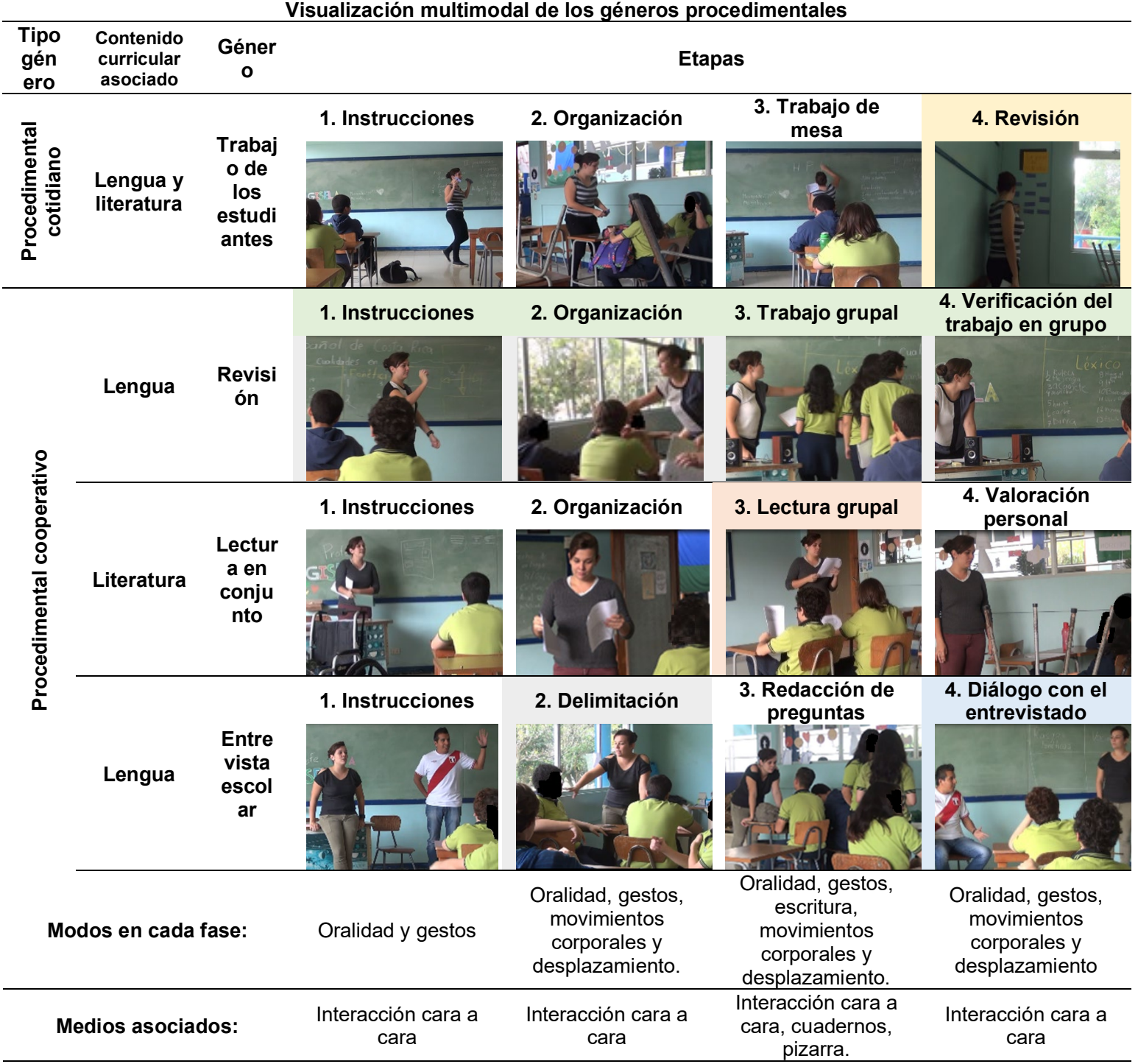

Fuente: elaboración propia con fotografías de archivo personal (2021).

\subsection{Géneros informativos: exposición docente}

El género exposición docente se definió como informativo y se registró tanto para contenidos curriculares de lengua como de literatura. Según los hallazgos, constituye un patrón recurrente, y en especial relevante, para modelar contenidos con los que la audiencia está poco familiarizada, independientemente de la naturaleza curricular de estos últimos. Este rasgo explica su versatilidad y empleo en función de discursos de variada naturaleza.

Por otra parte, los procesos de construcción del discurso escolar de este género informativo guardan similitudes epistémicas con los géneros relato histórico y explicación de las causas (Manghi, 2013a, Manghi, Badillo y Villacura, 2014, Manghi y Badillo, 2015), por 
cuanto las tres interacciones motivan la interpretación de los conocimientos desde las convenciones científicas propias de cada disciplina.

El género exposición docente se organiza en las siguientes etapas típicas (Tabla 7):

Tabla 7

Visualización multimodal del género informativo

\begin{tabular}{|c|c|c|c|c|c|}
\hline $\begin{array}{l}\text { Tipo de } \\
\text { género }\end{array}$ & Género & & Etapas & & $\begin{array}{l}\text { Contenido } \\
\text { curricular } \\
\text { asociado }\end{array}$ \\
\hline \multirow[b]{2}{*}{ Informativo } & \multirow[b]{2}{*}{$\begin{array}{l}\text { Exposición } \\
\text { docente }\end{array}$} & $\begin{array}{l}\text { 1. Conocimientos } \\
\text { previos }\end{array}$ & $\begin{array}{l}\text { 2. Presentación del } \\
\text { contenido }\end{array}$ & 3. Síntesis & \multirow{4}{*}{$\begin{array}{l}\text { Lengua y } \\
\text { literatura }\end{array}$} \\
\hline & & & & & \\
\hline \multicolumn{2}{|c|}{ Modos en cada fase: } & $\begin{array}{l}\text { Oralidad, gestos, } \\
\text { movimientos corporales } \\
\text { y desplazamiento }\end{array}$ & $\begin{array}{c}\text { Oralidad, gestos, } \\
\text { escritura, movimientos } \\
\text { corporales y } \\
\text { desplazamiento. } \\
\end{array}$ & $\begin{array}{c}\text { Oralidad, gestos, } \\
\text { movimientos corporales } \\
\text { y desplazamiento }\end{array}$ & \\
\hline \multicolumn{2}{|c|}{ Medios asociados: } & Interacción cara a cara & $\begin{array}{c}\text { Interacción cara a cara, } \\
\text { cuadernos, pizarra. }\end{array}$ & Interacción cara a cara & \\
\hline
\end{tabular}

Fuente: elaboración propia con fotografías de archivo personal (2021)

\subsection{Funciones representacionales, interpersonales y textuales}

En los géneros procedimentales, las funciones representacionales están vinculadas con las prácticas de reelaboración discusiva. Cada fase cumple funciones distintas y progresivas para la ejecución de estas últimas y, por lo tanto, para la construcción del contenido curricular. Así, le confieren al procedimiento su sentido de realización gradual y completable (Lemke, 1990), y resignifican el contenido al finalizar la interacción.

La fase de instrucciones cumple la función de definir el procedimiento, la secuencia de etapas para su realización, los resultados o productos esperados, así como los parámetros de evaluación de estos últimos. En el caso de la entrevista escolar y la lectura en conjunto, esta fase delimita interpersonalmente el procedimiento desde el inicio del género, ya que anticipa la situación comunicativa de lectura grupal y entrevista, a partir de las cuales orienta el posterior trabajo con el texto literario y la planificación dialógica.

La fase de organización se encarga de definir los modos y medios semióticos requeridos para las prácticas de reelaboración discursiva, y las condiciones que aseguren una ejecución exitosa del procedimiento. En el caso de la entrevista escolar, además de las funciones anteriores, esta fase cumple con el propósito de explorar ejes temáticos por abordar durante la entrevista, previamente a su redacción escrita (ver Tabla 6, sombreado gris). Así, 
cumple una función adicional de delimitación, en la medida en que filtra los focos temáticos y orienta el diseño del intercambio comunicativo del estudiantado.

Pese a que esta función "delimitadora" está ausente de los demás procedimientos, este estudio sostiene que, en esencia, constituye un mecanismo - de tipo discursivo - para organizar la realización posterior del género y que es exigida por el producto final esperado. Es decir, esta función responde a que el género escolar "entrevista" requiere, como procedimiento, planificar un género discursivo de la misma naturaleza. Por este motivo, se decidió conservar su nombre como “organización” y enmarcar dentro de ella dicha particularidad funcional.

En las fases nucleares, la función representacional consiste en reinterpretar el contenido curricular mediante la ejecución de las prácticas de reelaboración discursiva. Esta reinterpretación del contenido es el rasgo funcional común a todos los núcleos genéricos, independientemente de la actividad de mediación. Es decir, en todos los procedimientos, las ATA nucleares son distintas (resolver una práctica escrita, leer un texto literario, redactar preguntas para una entrevista...); no obstante, guardan en común el hecho de desarrollar la mayor parte la creación semiótica, precisamente, en respuesta a las prácticas de reelaboración asignadas.

Las últimas etapas se caracterizan por desempeñar una función representacional evaluativa, la cual consiste valorar los significados elaborados en la fase nuclear, mediante la revisión de los resultados obtenidos en las prácticas de reelaboración discursiva y su retroalimentación. En el caso del género trabajo de los estudiantes, en este punto se inserta el género revisión, el cual evalúa los significados del núcleo mediante la ejecución de un nuevo procedimiento (ver Tabla 6, sombreado amarillo).

En el género entrevista escolar, esta metafunción evaluativa adquiere un matiz particular, ya que, es en esta última etapa, donde finalmente se lleva a cabo el intercambio dialógico con el entrevistado; por lo que la ejecución de la entrevista es simultánea a su evaluación (ver Tabla 6, sombreado azul). Este es un rasgo exclusivo, que lo diferencia de otros procedimientos cooperativos. Además, puede interpretarse como una extensión del núcleo hacia la última fase del género - su puesta en escena -.

En el género informativo, la función representacional está permeada por el uso de la estructura de actividad Ilamada "diálogo tripartito" (Lemke, 1990). Esta consiste en una dinámica de preguntas y respuestas con la docente, mediante la cual el estudiantado participa 
en la modelación del contenido curricular, ya sea, al proveer información inicial sobre el fenómeno o al reelaborar este último en sus propios términos, al finalizar la interacción.

El uso de esta estructura implica que el género construye multifocalmente el contenido, en algunas etapas desde la perspectiva de la docente y, en otras, del estudiantado. Además, la interpretación de cada agente se entreteje con la que realiza su contraparte, en las demás fases. Como resultado, el contenido curricular se significa de manera diversa e integrada al finalizar el género.

La fase de conocimientos previos construye una representación inicial del fenómeno desde la perspectiva del estudiantado. Esta funciona como la base - la plataforma conceptual -, que guía la modelación del contenido curricular en las etapas posteriores. La caracterización que se efectúa en esta etapa no pretende ser "sistemática ni científica" - en el sentido de un discurso especializado -, sino que se compone de rasgos aleatorios, asociados al fenómeno, siempre y cuando sean relevantes para el estudiantado.

La presentación del contenido se encarga de reconstruir la entidad - según fue elaborada en la etapa anterior -, desde la perspectiva de la docente, a partir de una caracterización validada científicamente de sus rasgos constituyentes. Esta etapa define el fenómeno desde el discurso aprobado por la ciencia, es decir, establece su versión autorizada y con las características que le asocia la comunidad de especialistas. La naturaleza "científica" de esta etapa es fundamental y constituye una diferencia representacional importante con la fase previa.

Finalmente, la fase de síntesis resume la entidad de forma breve y panorámica, mediante una recapitulación, por parte del estudiantado, de los rasgos fundamentales expuestos por la profesora. Esta etapa constituye el cierre de la exposición sobre el fenómeno - la síntesis de todos los atributos que lo componen - y consolida la representación científica de este último. Además, verifica la asociación entre los conocimientos iniciales y el discurso científico provisto.

En cuanto las funciones interpersonales, tanto los géneros procedimentales como el informativo, muestran un patrón en el que la docente y el estudiantado alternan su protagonismo como agentes en las distintas etapas, debido a razones pedagógicas y epistémicas. En los procedimentales, la alternancia responde al papel de la docente como diseñadora de la interacción y a la naturaleza procesual de los géneros, la cual requiere la mentoría de un experto que oriente la secuencia de actividades (Martin y Rose, 2008). En el 
género informativo, la alternancia es motivada por el diálogo tripartito (Lemke, 1990) y los cambios en el agente desde cuya perspectiva se representan, en cada fase, las entidades.

En los géneros procedimentales, la etapa de instrucciones y organización legitiman la autoridad de la docente y le confieren mayor protagonismo: la primera, por cuanto esta define el procedimiento y conduce su ejecución; la segunda, puesto que la profesora determina la modalidad de trabajo (individual o grupal), los términos para la participación del estudiantado, así como los criterios para el empleo de los modos y medios semióticos. Por tanto, establece sus posibilidades intervención y representación del contenido curricular.

En los procedimientos cooperativos, la función interpersonal de la fase de organización responde a la naturaleza colectiva de estos géneros, la cual requiere la contribución de diversos participantes. Por este motivo, a diferencia del género trabajo del estudiantado, esta fase asume la modalidad grupal de trabajo como premisa y se emplea, específicamente, para distribuir los turnos de intervención, recursos y aspectos de las prácticas de relaboración discursiva que le corresponden a cada miembro.

Por otra parte, en el caso concreto de la entrevista escolar, como se señaló antes, la configuración interpersonal del género es definida tempranamente, desde la fase de "instrucciones", al establecer la situación comunicativa de entrevista. Así, asociada a su metafunción representacional, la fase de instrucciones desempeña una metafunción interpersonal específica: establecer las relaciones entre los participantes a modo de intercambio y prever la naturaleza dialógica de las intervenciones.

Las fases nucleares invierten el protagonismo de las fases anteriores y convierten al estudiantado en el agente de la interacción, mediante su involucramiento activo, de forma individual o grupal, en la ejecución de las prácticas de reelaboración discursiva. La manifestación de este protagonismo está influida por la naturaleza cotidiana o cooperativa del género y la modalidad de trabajo predominante. Así, el trabajo del estudiantado suele favorecer la actividad individual y, los procedimientos cooperativos, la participación colectiva.

Las últimas fases de los géneros procedimentales retornan el protagonismo a la docente y legitiman su posición de autoridad epistémica, debido la función representacional evaluativa de dicha fase. Dado que esta etapa se encarga de valorar los significados elaborados en la fase nuclear, la profesora adquiere una relevancia particular, al ser quien realiza observaciones sobre los aprendizajes y el conocimiento científico. Esta legitimidad es especialmente evidente el trabajo del estudiantado, donde no solo la metafunción, sino las 
ATA principales, suelen ser dinámicas para brindar retroalimentación individualizada a cada estudiante.

Ahora bien, para la evaluación, los tres géneros cooperativos se distinguen por efectuar una puesta en común de los resultados obtenidos en las prácticas de reelaboración discursiva (Tabla 6). Por este motivo, simultáneamente a la legitimación de la docente, quien evalúa los significados construidos, la metafunción interpersonal de estos géneros dota de un alto protagonismo al estudiantado, mediante el intercambio de sus interpretaciones y la construcción colectiva del contenido. Esta es una diferencia importante respecto del trabajo del estudiantado y se encuentra asociada a su naturaleza cooperativa.

En el caso del género informativo, la función interpersonal está relacionada con el uso de la estructura de actividad de diálogo tripartito (Lemke, 1990) y a los patrones de alternancia en los agentes. La etapa de conocimientos previos atribuye el protagonismo al estudiantado, quien construye la primera aproximación del fenómeno desde sus conocimientos y experiencia. Su centralidad en la modelación del contenido constituye una primera diferencia respecto de los géneros procedimentales, cuyas fases iniciales de instrucciones y organización priorizan a la docente como conductora de los procedimientos

En la fase de presentación del contenido, el protagonismo pasa a la docente, quien retoma la construcción del fenómeno y la enriquece desde una perspectiva científica. Esta es una segunda diferencia entre el género informativo y los procedimentales. Si bien en ambos el núcleo concentra la modelación del contenido; en estos últimos, el estudiantado es el agente principal, mediante la resolución de las prácticas de reelaboración discursiva. Por el contrario, en el género informativo, la profesora adopta este papel, al ejercer como autoridad epistémica que dirige la conceptualización del fenómeno.

En la fase de síntesis, por un lado, el estudiantado asume el protagonismo, al condensar, en sus propias palabras, los rasgos más relevantes de la entidad y proveer una recapitulación sistemática sobre esta. Por otro lado, la docente evalúa esta caracterización final del fenómeno, a la cual realiza ajustes de carácter científico. Este constituye un punto en común con las fases finales de los géneros procedimentales, en las que el protagonismo del estudiantado coexiste con la autoridad epistémica de la profesora.

Cabe destacar que la presencia del diálogo tripartito se registró, previamente, en la clase de historia, en la etapa "dinámica de preguntas y respuestas en torno a las causas" del género explicación de las causas (Manghi, 2013a) y en biología, en la fase "dinámica de preguntas y respuestas para construir la definición”, del género definición (Manghi y Córdova, 2011, 
Manghi, 2013a). Si bien difieren ligeramente en sus funciones representacionales, estas fases de preguntas y respuestas cumplen funciones interpersonales y finalidades epistémicas comunes con la clase de español, a saber: construir de manera interactiva el significado y enseñar al estudiantado las formas de razonamiento convencionales de cada área del conocimiento (Manghi, 2013a).

En cuanto a las funciones textuales, estas se encuentran altamente relacionadas con las funciones representacionales de cada fase. En los géneros procedimentales, las relaciones de cohesión y coherencia entre etapas se establecen según la ejecución lógica y procesual de las prácticas de reelaboración discursiva; en el informativo, de acuerdo con la construcción de la entidad semiótica y el aporte de cada fase y agente en su modelación. Como resultado, en ambos casos, las etapas enlazan bidireccionalmente unas con otras, en torno la fase nuclear la interacción.

En los géneros procedimentales, las instrucciones anticipan la organización, establecen los criterios para la ejecución de las actividades posteriores, las funciones de cada etapa y las relaciones entre ellas (Figura 1. Flecha 1). La organización retoma las instrucciones anteriores y prepara las condiciones para efectuar el procedimiento, en coherencia sus fases y tareas de representación (Figura 1. Flecha 2). Al mismo

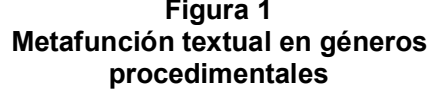
procedimentales

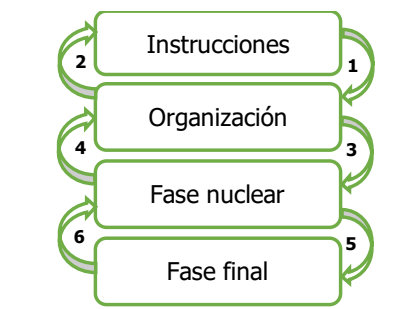

Fuente: elaboración propia (2021). tiempo, anticipa el núcleo del género, al proveer los recursos materiales y criterios de organización para resolver las prácticas de reelaboración discursiva (Figura 1. Flecha 3).

Las fases nucleares retoman la organización y ejecutan las prácticas de reelaboración designadas en las instrucciones, en coherencia con sus lineamientos, organización interpersonal, modos y medios semióticos establecidos (Figura 1. Flecha 4). De forma simultánea, anticipan las últimas fases genéricas, al crear los significados que se evaluarán en este punto, epistémica y procedimentalmente (Figura 1. Flecha 5). Las últimas etapas retoman los contenidos construidos en la fases nucleares, verifican la ejecución del procedimiento y los productos o resultados obtenidos (Figura 1. Flecha 6). Llegado a este punto, el género se da por terminado.

En los procedimientos cooperativos, la función textual permite distinguir el género lectura en conjunto, de la entrevista escolar y la revisión. Puesto que la lectura es colectiva, es necesario designar, constantemente, a los miembros del estudiantado que deben leer y las 
secciones del texto que les corresponden. Por este motivo, el género instancia las fases de organización y la etapa nuclear, de manera cíclica, en el siguiente patrón: asignación de turno, lectura; asignación de turno, lectura... hasta terminar el texto (ver Tabla 6, sombreado naranja). En contraste, en los géneros de revisión y entrevista escolar, las fases de organización y delimitación se instancian una única vez, tras las cuales da inicio el núcleo del género.

En el género informativo, la etapa de conocimientos previos modela la caracterización inicial del fenómeno y sugiere los aspectos pertinentes para su reconceptualización posterior (Figura 2. Flecha 1). La presentación del contenido retoma la construcción previa y reelabora el fenómeno con los significados que provee el discurso científico (Figura 2. Flecha 2). Además, esta fase redefine la entidad propuesta y construye una nueva, la cual funciona, a su vez, como material conceptual por evaluar en la etapa de síntesis (Figura 2. Fecha 3). Esta última se remite al fenómeno según fue construido en el núcleo y consolida la nueva caracterización, mediante una recopilación y valoración del discurso

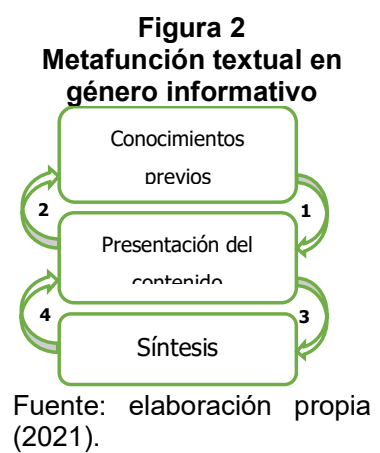
(2021). científico (Figura 2. Flecha 4). La fase de síntesis culmina la modelación del fenómeno y constituye el cierre del género.

En relación con los estudios anteriores, los hallazgos de este apartado sugieren que existe poca regularidad entre los géneros registrados en las clases de español, historia, biología y matemática. Esta divergencia parece indicar que son específicos para cada asignatura y se han especializado para instanciar los discursos de sus respectivas disciplinas. A su vez, esta especialización podría prevenir que se repitan en campos disciplinares distintos. Como resultado, los géneros aquí identificados - trabajo del estudiantado, revisión, lectura en conjunto, entrevista escolar y exposición docente - parecen ser exclusivos de la clase de español, pues aún no se han registrado durante la mediación de otras disciplinas.

Pese a esta especificidad disciplinar de las interacciones, los resultados sugieren una mayor cercanía en los procesos de mediación de las clases de historia y español, que entre esta última, matemática y biología. El género relato histórico en historia (Manghi, 2013a, Manghi, Badillo y Villacura, 2014, Manghi y Badillo, 2015) coincide respecto de los modos y sus funciones semióticas con el género exposición docente en español. Por su parte, la explicación de las causas en historia (Manghi, 2013a, Manghi, Badillo y Villacura, 2014, Manghi y Badillo, 2015) coincide con las interacciones de diálogo tripartito en español y el género definición en biología (Manghi, 2013a). Finalmente, el género procedimental en historia 
(Manghi, Badillo y Villacura, 2014) despliega funciones comunes con la categoría de géneros procedimentales en la clase de español.

\subsection{Modos y medios semióticos asociados a las etapas genéricas}

De acuerdo con los resultados, en primer lugar, los significados que permite articular un modo no se pueden disociar de los géneros y fases en que se insertan, ya que provienen de su empleo dentro de interacciones pedagógicas (Kress et al., 2001; van Leeuwen, 2005). En segundo lugar, la función de cada modo constituye un punto intermedio entre las funciones de cada fase (Kress, 2010) y el propio potencial semiótico (van Leeuwen, 2005). Es decir, los modos adoptan las funciones de las etapas que los enmarcan y las modelan según sus alcances de representación, al servicio de objetivos pedagógicos específicos. Por las razones anteriores - su uso pedagógico contextualizado y asociación con etapas específicas -, los resultados se presentan tras haber descrito los géneros.

La Tabla 6 y la Tabla 7 revelan la articulación modal asociada típicamente con cada etapa. Por motivos de espacio, en este artículo, la explicación se restringirá al modo que, en cada fase, adoptó la mayor "predominancia semiótica". Esta última se determinó por ser aquel sobre el cual recae la mayor acción semiótica (Kress, 2010), por parte del participante cuyo protagonismo es más destacado en dicha etapa (ver funciones interpersonales). Para cada modo predominante, el análisis procura interconectar las funciones representacionales, interpersonales y textuales.

En los géneros procedimentales, la etapa de instrucciones registra una estrecha asociación con la oralidad y los gestos como modos, los cuales se utilizaron en todos los casos. La oralidad se emplea, fundamentalmente, para especificar y estructurar los procedimientos. De forma simultánea, los gestos definen, deícticamente, los recursos materiales disponibles en el aula para su ejecución. Como se ha insistido antes, la articulación de estos modos refuerza la autoridad de la docente, por cuanto conducen el trabajo e intervenciones del estudiando. Además, da inicio a la ejecución del género, y vincula este último con los medios semióticos y la materialidad del aula donde toma lugar.

La etapa de organización revela un vínculo estrecho con los movimientos corporales y desplazamiento como modos; los cuales parecen ser típicos de esta última. Los primeros proveen al estudiantado los medios semióticos requeridos para las prácticas de reelaboración discursiva. Así, concretan las condiciones materiales para la ejecución del procedimiento y capacitan a los participantes para su realización. 
Por su parte, el desplazamiento distribuye los medios semióticos a través del recorrido en el aula. En el caso del trabajo del estudiantado, se suelen brindar los mismos medios (v.g. fotocopias) a todos los participantes; en los géneros cooperativos, la distribución es selectiva, según los subgrupos o miembros, y asignaciones específicas que corresponden a cada uno. Interpersonalmente, este modo integra al estudiantado como una comunidad de agentes, al conectarlos través del espacio del aula y los medios semióticos.

En cuanto a la función textual, la articulación de movimientos corporales y desplazamiento establece relaciones de cohesión y coherencia discursiva entre las diversas etapas del género, al suministrar de los medios semióticos que se requerirán, trasversalmente, en todas ellas. Además, en coherencia con las funciones de la fase de organización, preparan las condiciones para las fases posteriores, de forma que sean aptas para la participación del estudiantado.

Las fases nucleares muestran una fuerte asociación con la escritura, la cual se registró en todos los casos como el modo predominante para la representación, independientemente del contenido curricular, la naturaleza cotidiana o cooperativa del procedimiento y la modalidad de trabajo individual o grupal. Por un lado, esta relación entre el núcleo y la escritura podría constituir un resabio de una concepción monomodal de la representación y comunicación, que - como se señaló antes - asume la lengua como recurso de significación por antonomasia (Kress, 2010).

Por otro lado, este vínculo entre núcleo y escritura podría verse justificado, en tanto permite alinear tres dimensiones relevantes de la mediación: el contexto pedagógico, la presencia de prácticas de reelaboración discursiva y la metafunción interpersonal de la fase. Así, en la etapa nuclear, el estudiantado asume el protagonismo y trabaja en la creación de su propio conocimiento al ejecutar o resolver, de forma escrita, las tareas de representación asignadas al inicio del género.

En cuanto la función representacional, la escritura resignifica el contenido curricular mediante las interpretaciones del estudiantado. Interpersonalmente, modela la representación interna del mundo, estados afectivos y procesos cognitivos de los participantes, en forma de una interpretación externa y delimitada conceptualmente sobre el contenido. En este proceso, la escritura retoma los contenidos de etapas o géneros anteriores - en el caso de la revisión y los reconstruye en esta fase nuclear, de acuerdo con los procedimientos asignados y criterios de las prácticas de reelaboración discursiva. 
El género lectura en conjunto requiere una mención especial, ya que, en su fase nuclear, la oralidad, los gestos y la escritura cumplen funciones particulares, que lo diferencian modalmente del resto de procedimientos cooperativos. En primer lugar, en este género, la escritura no materializa significados creados por el estudiantado, sino que se encuentra en el texto literario de partida. Es decir, su función representacional no radica en producir - como resultado - una reinterpretación del contenido, sino en proveer "el guion escrito" a partir del cual se desarrolla la interpretación oral y gestual del texto.

En estas circunstancias, los gestos y la oralidad son los modos a través de los cuales toma lugar la experiencia textual. La oralidad resignifica el contenido escrito del texto literario, mediante la interpretación verbal de los significados. Simultáneamente, los gestos asocian cargas afectivas, cognitivas y significados culturales al contenido literario, durante la interpretación oral.

Las últimas etapas de los géneros revelan la predominancia no de un modo, sino de una articulación multimodal típica conformada por escritura, oralidad y gestos. Esta se explica por el propósito evaluativo de la fase y el protagonismo compartido por la docente y estudiantado. La escritura provee las reinterpretaciones de los fenómenos creadas por el estudiantado. Así, le confiere protagonismo y da seguimiento a las prácticas de reelaboración en fases y géneros anteriores, por lo que otorga unidad a los significados construidos.

La oralidad permite al estudiantado externar los significados escritos, mediante las interacciones verbales entre participantes. Así, afirma, ante la docente y el grupo de pares, su reinterpretación sobre el contenido. Por otra parte, la oralidad permite a la docente emitir valoraciones y reorientar las interpretaciones del estudiantado desde su autoridad como especialista. Textualmente, en ambos casos, este modo integra los significados construidos por los diversos agentes, a través de sus interacciones dialógicas y la articulación de sus aportes semióticos.

Finalmente, los gestos redimensionan los resultados del estudiantado y las valoraciones de la profesora, al concretar aspectos culturales, afectivos y cognitivos ausentes en su representación escrita y verbal. Interpersonalmente, reelaboran de forma individual el contenido, durante la intervención de cada participante. Textualmente, articulan los resultados escritos con su modelación verbal, durante la exposición de los hallazgos e interacciones entre participantes.

En el género informativo, las fases de conocimientos previos y síntesis mostraron la prevalencia de la oralidad y los gestos como modos. Esta asociación se debe al empleo 
de la estructura de actividad de diálogo tripartito (Lemke, 1990), la cual acentúa la interacción cara a cara y la modelación sincrónica, verbal y accional de los fenómenos por parte del estudiantado. Este hallazgo difiere, modalmente, de las etapas de preguntas y respuestas en historia y biología, las cuales emplean articulaciones de oralidad y gestos, junto con recursos gráficos (Manghi, 2013a; Manghi y Córdova, 2011).

La presentación del contenido reveló diferencias modales respecto de las fases anteriores, a raíz del protagonismo de la docente y al uso de diferentes recursos de apoyo para modelar las entidades. En esta fase, la profesora puede recurrir a imágenes, mapas conceptuales o diagramas, entre otros, para construir el contenido curricular (ver Tabla 7, sombreado amarillo), lo cual supone la selección de ciertos modos sobre otros y el despliegue de ciertas funciones acordes a su uso entrelazado. En consecuencia, en esta etapa, las articulaciones multimodales pueden ser muy diversas y numerosas, y están directamente relacionadas con las decisiones del profesorado sobre los recursos oportunos para la mediación, su disponibilidad y los medios semióticos utilizados (Kress et al., 2001).

Por los motivos anteriores, la fase nuclear de este género se caracterizó por reunir la mayor diversidad y complejidad semiótica, debido a la variedad de modos susceptibles de emplearse a fin de modelar los fenómenos y a la delicada articulación de sus funciones, que convergen en función de este objetivo pedagógico. Esta complejidad se registró, previamente, en los géneros relato histórico y definiciones (Manghi, 2013a), los cuales emplearon una amplia gama de recursos gráficos, lingüísticos y accionales para articular el conocimiento temporal con espacial y modelar, visualmente, narrativas de acontecimientos históricos y procesos naturales.

Debido a su diversidad y complejidad, un análisis detallado de los hallazgos para esta fase nuclear excede los propósitos de este trabajo. Para facilitar su exposición, los resultados se organizaron en tres escenarios, precisamente, según los recursos de apoyo empleados: casos en los que se diseñan, sincrónicamente, formas gráficas de representación para modelar la entidad semiótica en la pizarra; casos en que se modelan significados, escritos previamente, para construir la entidad semiótica y el uso de mapas, particularmente, para modelar la entidad semiótica. El detalle de los modos implicados en cada escenario y sus funciones se puede revisar en Flores (2019).

Los hallazgos de este apartado permitieron identificar modos semióticos comunes a los géneros de las clases de español, historia (Manghi, 2013a; Manghi y Badillo, 2015; Manghi, Badillo y Villacura, 2014), biología (Manghi, 2013a; Manghi y Córdova, 2011) y matemática 
(Manghi, 2013c; Manghi, 2010); cuya aparición responde a la materialidad del cuerpo y a los medios semióticos inmediatamente disponibles en el salón de clases. Estos modos son: 1. la oralidad, realizada a través del apartado fonador, 2. la escritura, instanciada en el pizarrón, 3. recursos gráficos (Lemke, 1998) como dibujos, esquemas, diagramas, tablas o mapas, que pueden ser modelados contingentemente en la pizarra y 4 . los gestos, que toman lugar en el cuerpo del profesorado y estudiantado. El sistema visual (Kress et al., 2001) reveló la mayor diversidad, pues los recursos gráficos varían según las convenciones de cada disciplina, contexto escolar y contenido curricular.

A la vez, es posible sugerir algunos usos generales de los modos semióticos anteriores, comunes a las cuatro clases. La oralidad estructura en términos globales el desarrollo de los géneros, explica los términos para la construcción del contenido curricular e interviene en la recontextualización pedagógica de los discursos (Bernstein, 1990). La escritura se emplea para generar productos e interpretaciones semióticas por parte del estudiantado, formular de manera concisa metáforas gramaticales, representar la organización taxonómica de las entidades o relaciones entre los fenómenos representados, etiquetar los recursos visuales en correlación con la oralidad y focalizar las nomenclaturas técnicas que denominan los conceptos científicos (Manghi, 2013a; Manghi, 2013c).

Los mapas, tablas, esquemas y los dibujos esquemáticos favorecen la representación integrada de significados temporales con espaciales, por cuanto construyen de manera paulatina los procesos, fenómenos y entidades descritas de forma oral, y las relaciones entre las partes con el todo (Lemke, 1998). Los gestos cumplen funciones deícticas, puesto que aclaran ubicaciones en el espacio y en las representaciones gráficas. Además, modelan significados topológicos que ni la oralidad ni la escritura - ni los gráficos mismos - son capaces de comunicar tan precisamente (Lemke, 1998).

Respecto de los medios semióticos, la interacción cara a cara y el uso de la pizarra destacan como superficies comunes a todos los géneros y mayoría de las etapas, tanto en las clases de historia (Manghi, 2013a; Manghi y Badillo, 2015; Manghi, Badillo y Villacura, 2014), biología (Manghi, 2013a; Manghi y Córdova, 2011), matemática (Manghi, 2013c; Manghi, 2010) como en español. Por este motivo, se consideran medios semióticos transversales a todas ellas. La relevancia semiótica de la interacción cara a cara está estrechamente ligada con la oralidad y los gestos, a los cuales sirve como superficie de realización. La importancia del uso de la pizarra se asocia con el empleo de números, simbolismo matemático, escritura, 
listados, mapas, tablas y esquemas como recursos gráficos, los cuales se construyen de forma gradual y paralela a los procedimientos y explicaciones orales y gestuales.

Si bien el libro de texto escolar se utilizó como medio impreso de manera simultánea a las explicaciones orales en historia (Manghi, 2013a; Manghi y Badillo, 2015; Manghi, Badillo y Villacura, 2014) y biología (Manghi, 2013a; Manghi y Córdova, 2011), no se identificó para matemática (Manghi, 2013c; Manghi, 2010) ni español. Este es un hallazgo especialmente importante en este estudio, ya que desliga la clase de español, necesariamente, de un libro de texto como medio semiótico y sitúa la mirada en otras superficies y recursos materiales empleados durante la mediación. De igual manera, pese a que las dos primeras clases recurrieron a las proyecciones por computadora como medios tecnológicos - aunque en menores ocasiones -, estos medios no se registraron en matemática y español. Por las razones anteriores, ni el manual escolar y ni los recursos tecnológicos fueron incluidas como parte de los géneros en estas últimas.

\section{Conclusiones}

Los contenidos curriculares de la clase de español fueron instanciados, de forma recurrente, a través de cinco géneros: trabajo del estudiantado, revisión, lectura en conjunto, entrevista escolar y exposición docente. Este hallazgo permite concluir que, pese a que la mediación toma lugar a través de numerosas actividades didácticas distintas, a lo largo de diferentes sesiones, estas suelen corresponder a un número más reducido de configuraciones recurrentes de significado. La consistencia de uso de estas últimas y sus funciones representacionales, interpersonales y textuales respaldan su relevancia para la mediación.

En relación con los estudios anteriores, los géneros identificados parecen ser exclusivos de la clase de español, pues no se han registrado aun en otras disciplinas. Además, sus procesos de mediación del contenido curricular tienden a mostrar mayores similitudes con los géneros en la clase de historia, que con aquellos de las áreas de matemática y biología. Con todo, antes que reforzar separaciones epistémicas y disciplinares, este trabajo prefiere resaltar el hecho de que todas las clases comparten - en alguna medida - patrones de significación y usos modales; razón por la cual sugiere repensarlas en términos de un continuo de cercanía semiótico - discursiva.

Por último, si bien este trabajo no emite valoraciones sobre el desempeño de la docente estudiada, sugiere el análisis de los géneros como una herramienta valiosa, a fin de visibilizar estructuras típicas, funciones y posibles áreas de mejora en la actividad del profesorado e 
interacciones de clase con base en estas últimas. Este es un campo abierto para nuevas investigaciones sobre los géneros en contextos escolares, que empleen el enfoque multimodal para la evaluación de estrategias didácticas y la interacción de aula.

Respecto de los modos semióticos, la clase de español recurrió, predominantemente, a articulaciones de oralidad, escritura, gestos, movimientos corporales y desplazamiento en el espacio; y, en menor medida, al uso de recursos gráficos, principalmente, en el género exposición docente. En respuesta a estos modos, la interacción cara a cara, el uso de la pizarra y del espacio del aula destacaron como medios semióticos más frecuentes. Estos últimos, a su vez, resultaron ser comunes a las clases de historia, biología y matemática y español.

Los hallazgos anteriores llevan a sugerir que la naturaleza "típicamente lingüística", desde la cual se suele pensar la clase de español, puede deberse a que se abordan contenidos curriculares relacionados con la lengua y literatura, y no a que la mediación se realice, en exclusiva, mediante la lengua oral y escrita, pues se constató la presencia de otros modos. Precisamente, a la luz de los múltiples recursos involucrados en la mediación, este estudio sugiere repensar la clase de español no como "típicamente lingüística", sino modalmente diversa, es decir, articulada por múltiples modos semióticos, todos los cuales se entrelazan durante la mediación.

Ahora bien, vista desde otra perspectiva, esta naturaleza "típicamente lingüística" atribuida a la clase de español puede responder a que las fases nucleares de los géneros solicitan al estudiantado resolver las prácticas de reelaboración discursiva, principalmente, a través de la escritura. Esta asociación puede reforzar la idea de que el conocimiento se construye, principalmente, mediante el sistema lingüístico, lo cual robustece una percepción todavía monomodal de la comunicación y representación (Kress, 2010).

Esta centralidad de la lengua opaca la presencia de otros modos y medios que tanto la docente como el mismo estudiantado emplean durante los procesos de enseñanza y aprendizaje (Manghi, 2011). Por ello, se recomienda al profesorado incorporar, con mayor intencionalidad, otros modos de representación y comunicación en las fases nucleares de los géneros y - en general - visibilizar la multiplicidad de recursos que se articulan, de forma transversal, durante toda la mediación pedagógica.

Para finalizar, se debe recordar que esta investigación constituye un estudio de caso, por lo que sus resultados respecto de los géneros y modos no son generalizables. Aún más, este hecho refuerza la necesidad de nuevas investigaciones que cotejen sus resultados en distintos contextos y con diferentes participantes. Además, se reconoce abiertamente que 
estos hallazgos ofrecen una visión parcial, enfocada en la figura del docente y los procesos de enseñanza. Por lo tanto, son necesarias nuevas investigaciones que aborden la interacción entre profesorado y estudiantado, e incluso, sus implicaciones para el aprendizaje.

\section{Referencias}

Bernstein, Basil. (1990). Class, codes and control. Vol. 4: The structuring of pedagogic discourse. Londres, Reino Unido: Routledge

Bezemer, Jeff. y Kress, Gunther. (2008). Writing in multimodal texts: A social semiotic account of designs for learning. Written communication, 25(2), 166-195. doi: https://doi.org/10.1177/0741088307313177

Cope, Bill. y Kalantzis, Mary. (2009). A Grammar of Multimodality. International Journal of Learning, 16(2), 361- 426, doi: 10.18848/1447-9494/CGP/v16i02/46137

Flores, Carol. (2019). Modos semióticos y géneros en la enseñanza de lengua y literatura en secundaria: un estudio de caso (Tesis de maestría sin publicar). Universidad de Costa Rica: San José, Costa Rica. Recuperado de: http://hdl.handle.net/10669/83758

Halliday, Michael. (1982). El lenguaje como semiótica social. México, D.F.: Fondo de Cultura Económica.

Kress, Gunther. (2010). Multimodality: A social semiotic approach to contemporary communication. Londres, Reino Unido: Routledge.

Kress, Gunther. and Van Leeuwen, Teo. (2001). Multimodal discourse: The modes and media of contemporary communication. New York, USA: Hodder Arnold.

Kress Gunther., Jewitt, Carey., Ogborn, Jon. y Tsatsarelis, Charalampos. (2001). Multimodal teaching and learning: the rhetorics of the science classroom. Londres, Reino Unido: Continuum.

Lemke, Jay. (1990). Talking science: Language, learning, and values. New Jersey, USA: Ablex Publishing Corporation.

Lemke, Jay. (1998). Multiplying meaning: Visual and verbal semiotics in scientific text. En J. Martin y R. Veel (Eds.). Reading science: Critical and functional perspectives on discourses of science (pp. 87-113). Londres, Reino Unido: Routledge.

Manghi, Dominique y Córdova, Juan Pablo. (2011). Definiciones y explicaciones multimodales: potencial semiótico en la enseñanza de la biología en educación media multimodal. Logos: Revista de Lingüística, Filosofía y Literatura, 21(2), 17-39. Recuperado de https://revistas.userena.cl/index.php/logos/article/view/165

Manghi, Dominique. (2010). Recursos semióticos del profesor de matemática: funciones complementarias del habla y los gestos para la alfabetización científica escolar. Estudios pedagógicos 36(2), 99-115. doi http://dx.doi.org/10.4067/S0718-07052010000200006 
Manghi, Dominique. (2011). La perspectiva multimodal sobre la comunicación: desafíos y aportes para la enseñanza en el aula. Diálogos educativos, (22), 3-14. Recuperado de https://dialnet.unirioja.es/servlet/articulo?codigo=3931351

Manghi, Dominique. (2013a). Géneros en la enseñanza escolar: Configuraciones de significado en clases de historia y biología desde una perspectiva multimodal. Revista signos, 46(82), 236-257, doi: 10.4067/S0718-09342013000200004

Manghi, Dominique. (2013b). Representación y comunicación del conocimiento en Educación Media: análisis multimodal del discurso de materiales utilizados para la enseñanza escolar de la historia y de la biología. Onomázein, (27), 35-52. Recuperado de https://dialnet.unirioja.es/servlet/articulo?codigo=4419909

Manghi, Dominique. (2013c). La mediación del profesor especialista para la alfabetización semiótica en el aula de Matemática. En E. I. Moyano (Coord). Aprender ciencias y humanidades: una cuestión de lectura y escritura. Aportes para la construcción de un programa de inclusión social a través de la educación lingüística (pp.199-226). Los Polvorines: Universidad Nacional de General Sarmiento.

Manghi, Dominique. y Badillo, Carolina. (2015). Modos semióticos en el discurso pedagógico de historia: Potencial semiótico para la mediación en el aula escolar. Ikala, Revista de lenguaje $y$ cultura, 20(2), 157-172. Recuperado de http://www.redalyc.org/articulo.oa?id=255042625002

Manghi, Dominique., Badillo, Carolina. y Villacura, Paula. (2014). Alfabetización semiótica en clases de Historia: Estrategias de mediación desde un enfoque multimodal. Perfiles educativos, 36(146), $63-79 . \quad$ Recuperado de http://www.scielo.org.mx/scielo.php?script=sci arttextypid=S0185$\underline{26982014000400005 y \mid n g=e s y t \operatorname{lng}=e s}$

Martin, James. y Rose, David. (2008). Genre relations: mapping culture. Londres, Reino Unido: Equinox.

Quintana-Peña, Alberto. (2006). Metodología de investigación científica cualitativa. En A. Quintana y W. Montgomery (Eds.), Psicología: Tópicos de actualidad (pp. 47-84). Lima, Perú: Universidad Nacional Mayor de San Marcos. Recuperado de http://biblioteca.udgvirtual.udg.mx/jspui/handle/123456789/2724

Sánchez, Emilio., García, J. Ricardo., Rosales, Javier., de Sixte, Raquel., y Castellano, Nadezhna. (2008). Elementos para analizar la interacción entre estudiantes y profesores: ¿qué ocurre cuando se consideran diferentes dimensiones y diferentes unidades de análisis? Revista de Educación, 1(346), 105-136. Recuperado de https://www.researchgate.net/publication/277276766

Van Leeuwen, Teo. (2005). Introducing social semiotics. Londres, Reino Unido: Routledge. 
Revista indizada en

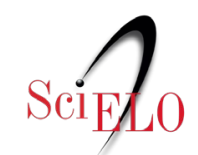
redalyc sy latindex

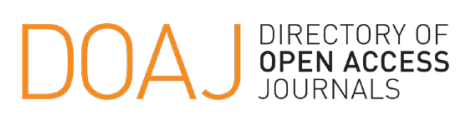

Distribuida en las bases de datos:

- Dialnet
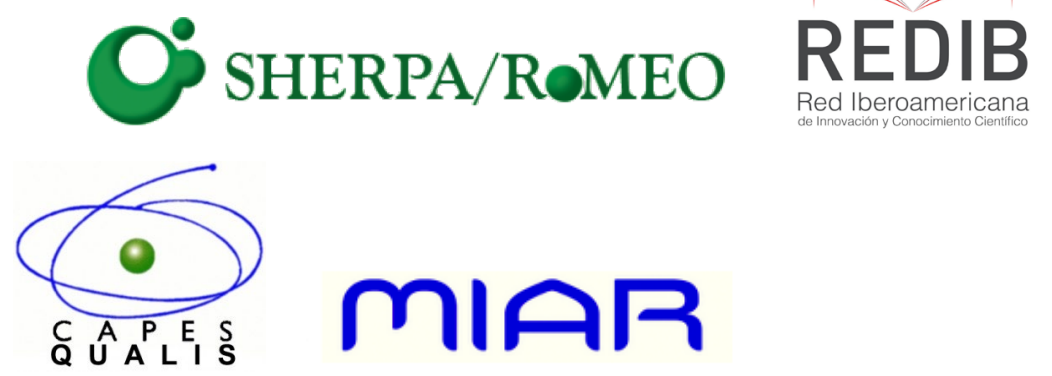

MIAR 